\title{
Displasia cemento óssea-florida tratada cirurgicamente: relato de caso
}

\author{
Florid cemento-osseous dysplasia surgically treated: a case report
}

Displasia cemento bono-florida cirugía tratada: presentación de caso

\begin{abstract}
Marcos Pablu Leite Sales Souza ${ }^{1 *}$, Islana de Souza Brito ${ }^{1}$, Ana Luiza Pontes de Oliveira1 ${ }^{1}$, Olívia Maria Guimarães Marroquim¹, Aurea Valéria de Melo Franco ${ }^{1}$, Fernanda Braga Peixoto.
\end{abstract}

\begin{abstract}
RESUMO
Objetivo: Relatar um caso clínico de Displasia Cemento Óssea Florida sintomática em uma mulher, identificando as características clínicas, histopatológicas e radiográficas. Detalhamento do Caso: Paciente de 60 anos de idade, Gênero feminino, melanoderma, portadora de hipertensão arterial que procurou a Clínica Escola de Odontologia de uma Instituição de ensino superior, queixando-se de que não conseguia tomar água e apresentava dores em sua face. $\mathrm{Na}$ história da doença observou-se a realização de exodontia há cerca de um ano, a qual evoluiu com dor e edema, observou-se no exame intrabucal a presença de um crescimento ósseo exposto em região de rebordo alveolar, fixo, localizado do lado esquerdo em região de molares inferiores, limites nítidos, superfície irregular, consistência pétrea, coloração amarelada com áreas pardacentas, considerando assim as hipóteses diagnósticas optando-se pela realização de biópsia incisional. Foi realizado um tratamento e a paciente está em acompanhamento através de exames clínicos e radiográficos. Considerações Finais: A Displasia Cemento-Óssea Florida (DCOF) é uma condição fibroso-óssea não neoplásica de origem desconhecida. Após o tratamento, a paciente está em acompanhamento, até o momento, não relatou sintomatologia dolorosa e os exames demonstraram ausência da (DCOF).
\end{abstract}

Palavras-chave: Patologia bucal, Displasia fibrosa óssea, Cemento dentário.

\begin{abstract}
Objective: To report a clinical case of symptomatic Florida cementum dysplasia in a woman, identifying the clinical, histopathological and radiographic characteristics. Case Details: 60-year-old female patient, melanoderma, with hypertension who came to the Dental School Clinic of a higher education institution, complaining that she was unable to drink water and had pain in her face. In the history of the disease, extraction was performed about a year ago, which evolved with pain and edema. In the intraoral examination, the presence of bone growth was observed in the region of a fixed alveolar ridge, located on the left side. In the region of lower molars, sharp limits, irregular surface, stone consistency, yellowish color with brownish areas, thus considering the diagnostic hypotheses, opting for incisional biopsy. A treatment was performed and the patient is being monitored through clinical and radiographic exams. Final Considerations: Florida Cement-Bone Dysplasia (DCOF) is a non-neoplastic fibrous-bone condition of unknown origin. After treatment, the patient is being monitored, so far, has not reported painful symptoms and the exams have shown absence of (DCOF).
\end{abstract}

Keywords: Oral pathology, Fibrous dysplasia of bone, Dental cementum.

\section{RESUMEN}

Objetivo: Reportar un caso clínico de displasia de cemento sintomática en Florida en una mujer, identificando las características clínicas, histopatológicas y radiográficas. Detalles del Caso: Paciente mujer de 60 años, melanoderma, con hipertensión que acudió a la Clínica Facultad de Odontología de una institución de educación superior, quejándose de no poder beber agua y con dolor en la cara. En la historia de la enfermedad se realizó extracción hace aproximadamente un año, la cual evolucionó con dolor y edema, en el examen intraoral se expuso crecimiento óseo en una región de reborde alveolar fijo ubicado en el lado izquierdo. en la región de los molares inferiores, límites marcados, superficie irregular, consistencia de piedra, color amarillento con áreas parduscas, considerando así las hipótesis diagnósticas, optando por la biopsia incisional. Se realizó un tratamiento y el paciente está siendo monitoreado mediante exámenes clínicos y radiográficos. Consideraciones Finales: La Displasia Cemento-Ósea Florida (DCOF) es una condición de hueso fibroso no neoplásico de origen desconocido, luego del tratamiento, el paciente está siendo monitoreado, hasta el momento no ha reportado síntomas dolorosos y los exámenes han mostrado ausencia de (DCOF).

Palabras clave: Patología bucal, Displasia fibrosa ósea, Cemento dental.

${ }^{1}$ Centro Universitário CESMAC (CESMAC), Maceió - AL. *E-mail: pabloensf11@gmail.com 


\section{INTRODUÇÃO}

As Lesões Fibro-Ósseas (LFO) constituem um grupo de lesões proliferativas reativas, displásicas ou neoplásicas que incluem: Displasia Fibrosa, Fibroma Ossificante e Displasia Cemento-Óssea (MACDONALD DS, 2015; COSTA FWG, et al., 2018). Há uma prevalência no gênero feminino, negras, sendo cerca de $90 \%$ dos casos diagnosticados em indivíduos entre a terceira e quarta década de vida. A Organização Mundial de Saúde - OMS (2017), classifica esta patologia através de parâmetros como: local da lesão, características clínicas, radiográficas e histopatológicas, além de sexo e idade, podendo apresentar-se de forma expansiva, levando a quadro de dores e possíveis infecções devido à escassa vascularização da área.

Esta representa o resultado de como os agentes etiopatogênicos agridem o nosso organismo e como os sistemas naturais de defesa reagem, não sendo diagnosticado apenas através de um exame de laboratório específico, pois, pode-se encontrar inúmeras patologias caracterizadas como sendo entidades fibro-ósseas, sendo necessário o uso de recursos clínicos e radiográficos para à confirmação da patologia, para que assim, possa-se chegar ao correto diagnóstico e um prognóstico favorável (PAPAIZ EG, et al., 2011).

A Displasia Cemento-Óssea (DCO), um dos tipos das LFO, apresenta-se como uma lesão benigna, caracterizada pela substituição do tecido ósseo normal por tecido conjuntivo fibroso celular e osso metaplásico. Esta patologia subdivide-se em três tipos: cemento-óssea periapical, quando restrita respectivamente na região anterior da mandíbula, cemento-óssea focal quando apresenta lesão única localizada em um quadrante na região posterior da mandíbula e cemento-óssea florida quando acomete bilateralmente os maxilares (AMARISTA-ROJAS FJ, et al., 2016; COSTA FWG, et al., 2018; DAVIET-NOUAL V, et al., 2017; LIMA ALC, et al., 2018; BRODY A, et al., 2019).

Dentro do grupo DCO, pode-se citar um subtipo intitulado Displasia Cemento-Óssea Florida (DCOF), que é uma condição fibroso-óssea não neoplásica de origem desconhecida, comumente assintomática. No entanto, alguns casos podem apresentar expansão óssea, dor, deformidade e formação de sequestros ósseos quando associada a infecções ou ainda à compressão ocasionada pela utilização de próteses mal adaptadas (AMARISTA-ROJAS FJ, et al., 2016; CARVALHO CHP, et al., 2012; SARMENTO DJS, et al., 2013; YILDIRIM E, et al., 2016).

Apesar da vaga etiologia da DCOF, alterações reativas como exodontias e alterações periodontais podem ser causa para a doença, através de exames clínicos, pode ser observado que essas formas de lesões na maioria das vezes não apresentam sintomatologia podendo ser encontradas através de exames radiográficos incidentais. Quando analisado as radiografias, o DCOF demonstra-se com aspectos de volumes de massas espeças e lobuladas com margens levemente irregulares, geralmente encontrada em várias áreas da mandíbula (SHAH S, et al., 2012).

Os pacientes que apresentam essa condição possuem sintomatologia, dos quais podem desencadear bastante incômodo e muito das vezes drenagem quase são associados aos processos terapêuticos à apresentação de massas escleróticas com calcificação no meio bucal, isto é, osteomielite de forma secundária (CAPELLA LRC e OLIVEIRA RJ, 2014). Em aspectos histológicos, essas formas de lesões são concebidas por osso trabecular anastomosado que dispõem segundo as linhas de pressão que atuam nesta área fazendo com que o osso esponjoso fique bastante resistente, porém leve e apresenta camadas de calcificações semelhantes ao cemento embebidas em matriz fibroblástica (CARVALHO CHP, et al., 2012).

Radiograficamente, a Displasia Cemento-Óssea Florida exibe massas de radiopacidade mista localizadas bilateralmente em região mandibular adjacentes aos ápices radiculares (AMARISTA-ROJAS FJ, et al., 2016; KATO CNAO, et al., 2018; SARMENTO DJS, et al., 2013; YILDIRIM E, et al., 2016). Com o amadurecimento, a lesão pode tornar-se radiograficamente mais radiopaca (DAVIET-NOUAL V, et al., 2017), devido a alterações na proporção entre tecido conjuntivo fibroso e material mineralizado (NEVILLE BW, et al., 2016; PEREIRA DL, 2016).

Ao exame histopatológico observa-se a presença de tecido conjuntivo fibroso, osso imaturo, e partículas semelhantes ao cemento e presença de infiltrado inflamatório quando associada a processos infecciosos. $O$ tratamento da DCO só é recomendado para os casos onde exista infecção local associada. Já nos casos assintomáticos, é válido à realização de avaliações periódicas clínicas e radiográficas. (AMARISTA-ROJAS FJ, et al., 2016; DAVIET-NOUAL V, et al., 2017; COSTA FWG, et al., 2018). 
Desta forma, o respectivo estudo possui como indispensável parâmetro, através de um relato de caso, descrever um caso de Displasia Cemento Óssea Florida sintomática, identificando as características clínicas, histopatológicas e radiográficas, demonstrando que o Cirurgião Dentista (CD) precisa compreender o dinamismo dessa patologia, sendo levado em consideração suas particularidades e o conhecimento cientifico sobre analise dos exames radiográficos e clínicos.

\section{DETALHAMENTO DO CASO}

Paciente do gênero feminino, melanoderma, 60 anos de idade, foi encaminhada para a clínica odontológica de uma Instituição de ensino superior, queixando-se de que "não conseguia tomar água e meu rosto está inchado". Na história da doença realização de uma exodontia há cerca de um ano, a qual evoluiu com dor e edema. Foi realizado tratamento antibiótico com Amoxicilina $875 \mathrm{mg}$, resultando na diminuição do edema e da sintomatologia dolorosa.

Quando questionada sobre a história médica, a paciente relatou ser portadora de hipertensão arterial sistêmica e estar sobre cuidados médicos, fazendo o uso do medicamento Losartana que é um fármaco da classe dos antagonistas dos receptores da angiotensina. Em que sua principal indicação é para tratamento de hipertensão arterial, sendo aplicado nessa paciente a cada 12 horas. Ao ser realizado o exame extrabucal, a paciente não apresentava nenhuma alteração digna de nota.

Durante o exame intrabucal foi observada a presença de um crescimento ósseo exposto em região de rebordo alveolar, fixo, localizado do lado esquerdo em região de molares inferiores, limites nítidos, superfície irregular, consistência pétrea, coloração amarelada com áreas pardacentas. A Figura 1 representa o aspecto inicial do quadro clínico da região acometida pela lesão.

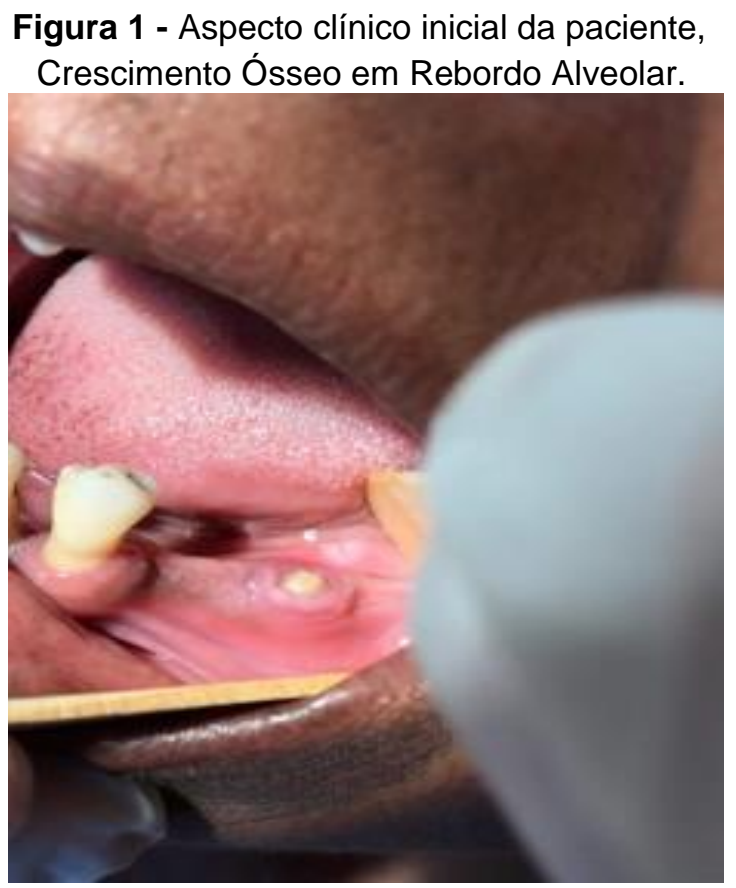

Fonte: Souza MPLS, et al., 2020.

A conduta inicial foi a realização da radiografia panorâmica, na qual foram observadas múltiplas áreas radiopacas distribuídas pelos quadrantes inferiores, tendo como principal hipótese clínica Displasia Cemento Óssea Florida, Doença de Paget no osso e Osteomielite Crônica Esclerosante Difusa. A Figura 2 apresenta a radiografia panorâmica do paciente com a respectiva patologia. Considerando as hipóteses diagnósticas, a localização e a extensão da lesão, optou-se pela realização de biópsia incisional. Inicialmente foi realizada anestesia local por meio da técnica supraperiosteal para bloqueio dos nervos lingual, bucal e alveolar inferior. O anestésico utilizado foi cloridrato de lidocaína 2\% com epinefrina 1:100.000 (ALPHACAINE $100^{\circledR}$, DFL, Rio de Janeiro, Brasil). 
Figura 2 - Múltiplas áreas Radiopacas distribuídas pelos quadrantes inferiores.

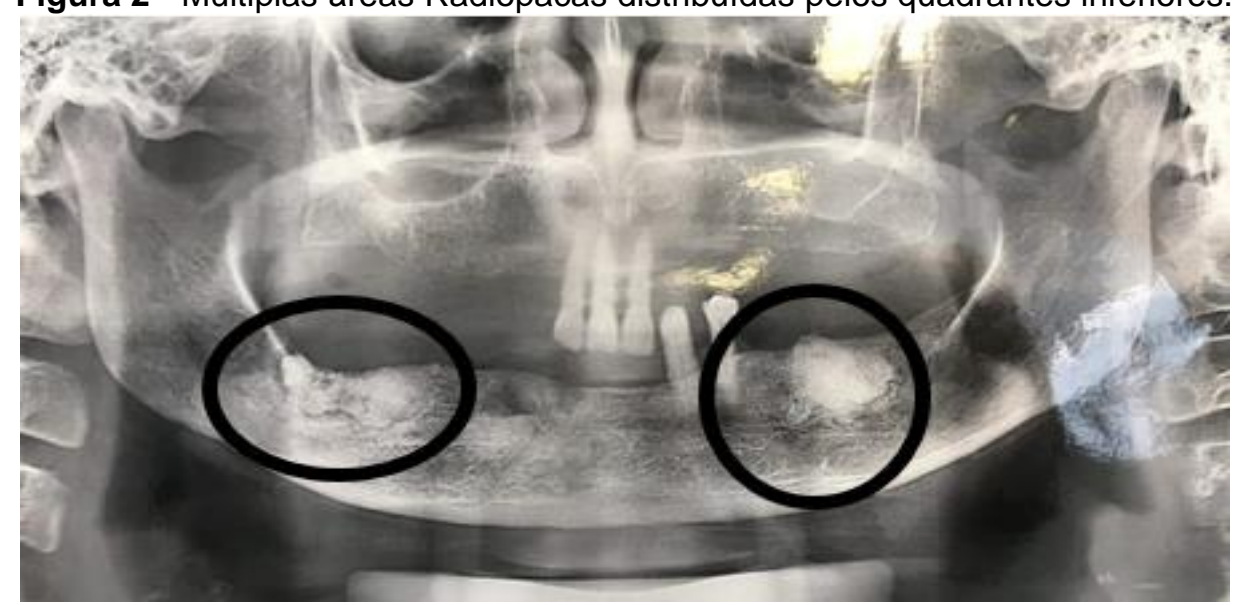

Fonte: Souza MPLS, et al., 2020.

Em seguida, foi realizada incisão intrasulcular mandibular com descolamento de retalho mucoperiósteo, utilizando bisturi lâmina 15 (Solidor, São Paulo, Brasil). Para osteotomia utilizou-se broca esférica 702 e 703 multilaminada (KG Sorensen, São Paulo, Brasil), sob irrigação com soro fisiológico. A lesão foi exposta, facilmente destacada do osso adjacente. À Figura 3 representa às etapas incisão e descolamento mucoperiósteo, respectivamente. Em seguida o retalho mucoperiósteo foi reposicionado e suturado com pontos isolados simples, utilizando fio de seda Ethicon ${ }^{\mathrm{TM}} 4.0$ milímetros (Johnson \& Johnson, São Paulo, Brasil). A Figura $3 \mathrm{C}$ apresenta a etapa de síntese da cirurgia. A peça cirúrgica foi fixada em solução de formaldeído a $10 \%$ e enviada ao laboratório da instituição para exame anatomopatológico e posterior análise histopatológica. A Figura $3 \mathrm{D}$ apresenta a peça cirúrgica.

Figura 3 - Procedimento de incisão intrasulcular mandibular com deslocamento de retalho mucoperiósteo.
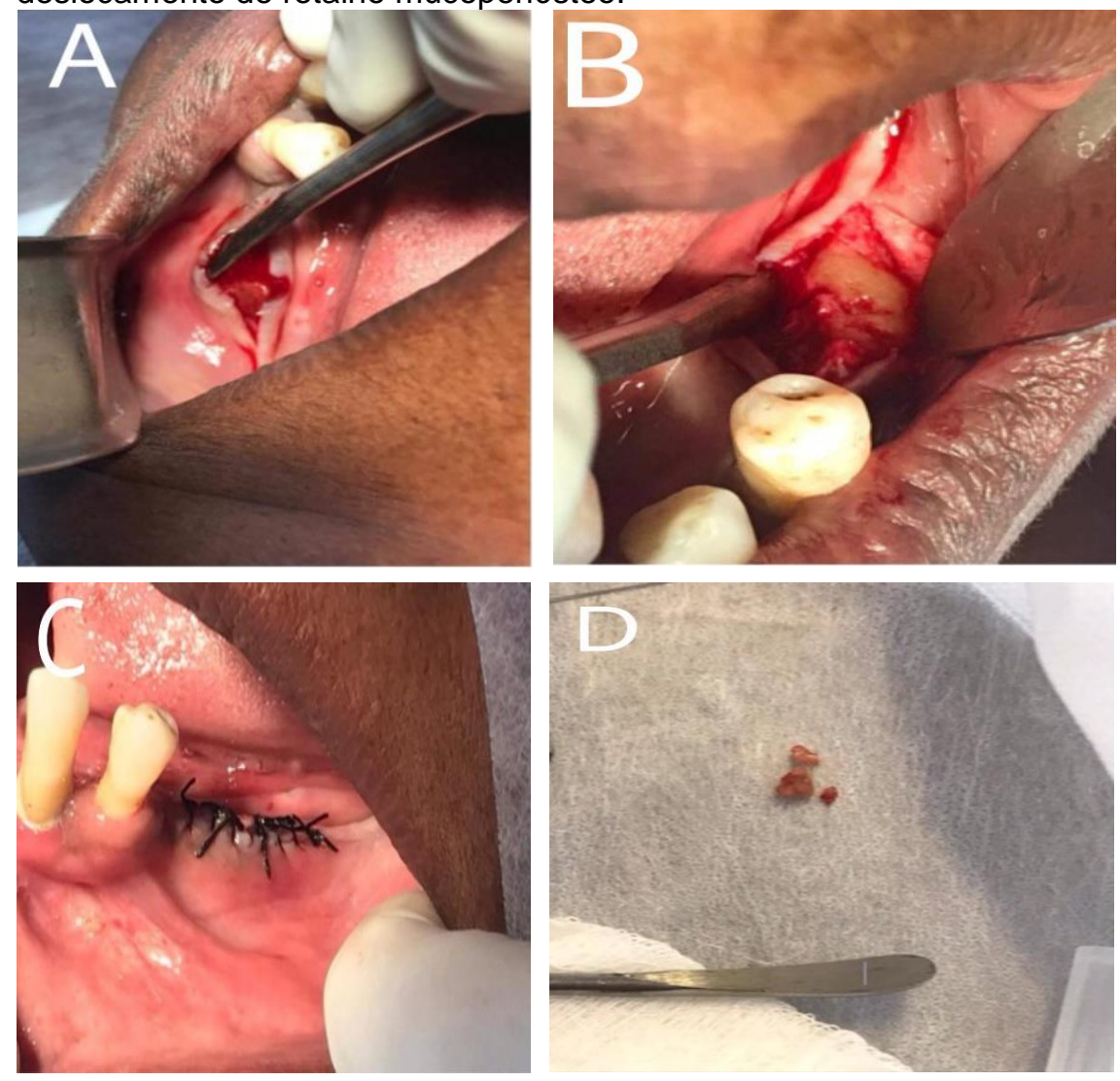

Legenda: A: Descolamento de retalho mucoperiósteo; B: Lesão exposta; C: Suturas em pontos simples; D: Peça cirúrgica.

Fonte: Souza MPLS, et al., 2020. 
O material recebido para exame histopatológico constava de 4 fragmentos de tecido duro, medindo em

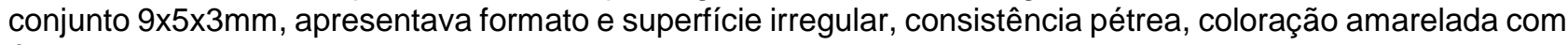
áreas pardacentas.

Os cortes histológicos corados em Hematoxilina-Eosina (HE) revelam fragmentos tecido mineralizado compatível com tecido ósseo compacto. Confirmando a hipótese diagnóstica de Displasia Cemento Óssea Florida. A Figura 4 representa os cortes histológicos nas aproximações 100x e 400x, respectivamente.

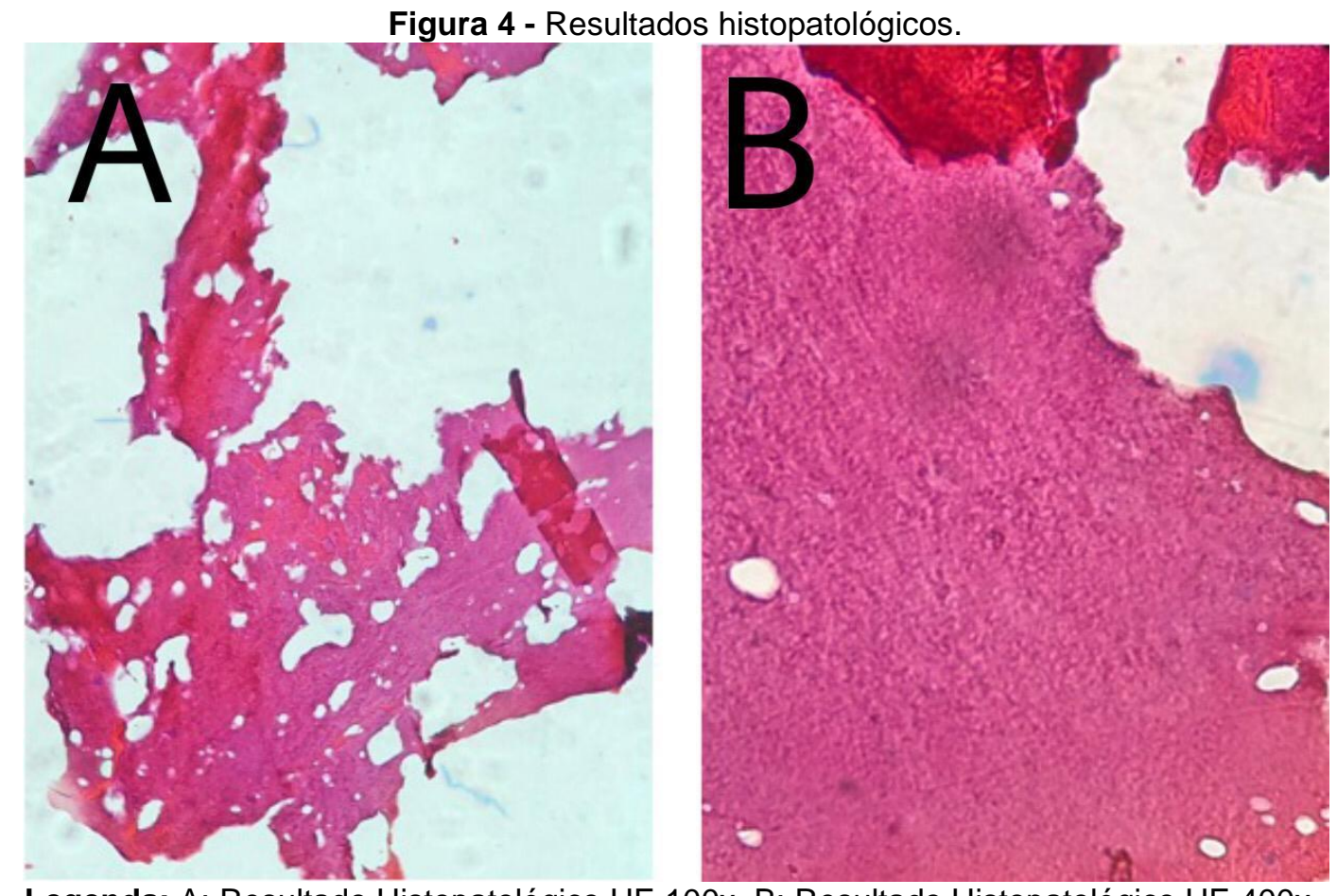

Legenda: A: Resultado Histopatológico HE 100x. B: Resultado Histopatológico HE 400x.

Fonte: Souza MPLS, et al., 2020.

Após o tratamento cirúrgico, indicado pela exposição óssea e sintomatologia dolorosa, a paciente está em acompanhamento através de exames clínicos e radiográficos e, até o momento, não relatou sintomatologia dolorosa. Sendo assim, pode-se considerar que a recuperação foi satisfatória (Figura 5).

Figura 5 - Proservação com 6 meses de acompanhamento pós operatório.

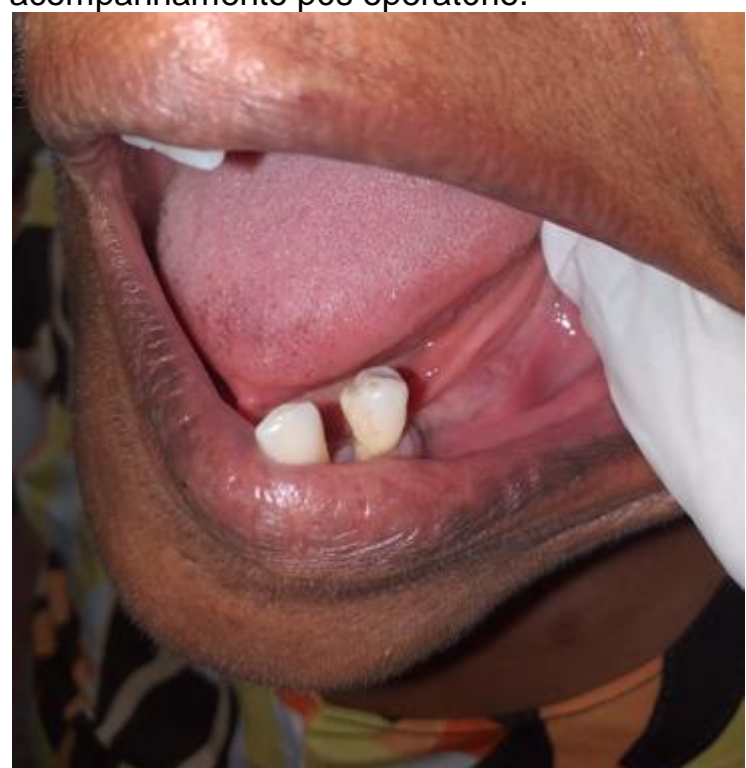

Fonte: Souza MPLS, et al., 2020 


\section{DISCUSSÃO}

A Displasia Cemento Óssea Florida (DCO) é uma condição fibro-óssea benigna, que se caracteriza pela substituição do tecido ósseo normal por tecido conjuntivo fibroso celular e osso metaplásico (DAVIET-NOUAL V, et al., 2017; YILDIRIM E, et al., 2016). Possui predileção pela raça negra e mulheres de meia idade, não tendo uma explicação precisa para esse fato (OLIVEIRA CNA, 2016; BRODY A, et al., 2019). No presente caso, constatamos a lesão em uma paciente melanoderma, do sexo feminino, de 60 anos de idade, tornando os fatos condizentes com o relatado na literatura.

Brody A, et al. (2019), demonstraram que em casos dos quais ocorrem as lesões de forma sintomática os encontrados em radiografias são percebidos de forma significativas e bastante aparente, assim como é demonstrado nesse estudo, já que a paciente possui como um dos principais motivos da ida a clínica Odontológica as dores que sentia e através do exame radiográfico e de imagem foi constatado a Displasia Cemento-Óssea Florida.

No presente estudo, a paciente descreveu dores de forma grave e algumas vezes moderada, sendo na maioria das vezes de forma intermitente, apenas do lado esquerdo da mandíbula, sendo essa apresentação específica coerente com osteomielite crônica que ocorre quando ela se apresenta de forma ativa por mais de seis semanas. Via de regra, o quadro acontece em decorrência de uma lesão aguda que não passou por um tratamento terapêutico de forma adequada e foi-se agravando lentamente, porém de forma continua e estão de acordo com os relatos anteriores de Souza Júnior EJC, et al (2008) e El-Naggar AK, et al. (2018), o que reafirma ainda mais a importância dos exames laboratoriais relacionado aos exames que complementam aos dados da anamnese e do exame físico para a confirmação das hipóteses diagnósticas e os possíveis tratamentos a serem realizados, buscando em todo tempo do tratamento associar as particularidades das sintomatologias, como presença de dor, expansão óssea, deformidade e sequestros ósseos, uma vez que esta pode-se apresentar assintomática (CASTRO TF, et al., 2017).

A avaliação imaginológica minuciosa é essencial no diagnóstico e posterior conduta terapêutica da DCOF, uma vez que esta possui como diagnóstico diferencial a presença de partículas semelhantes ao cemento, osso imaturo e tecido conjuntivo fibroso, o que torna dessemelhante de lesões como síndrome de Gardner (COSTA FWG, et al., 2018), osteomielite esclerosante difusa (AMARISTA-ROJAS FJ, et al., 2016; CASTRO TF, et al., 2017; COSTA FWG, et al., 2018) e doença de Paget (AMARISTA-ROJAS FJ, et al., 2016; CASTRO TF, et al., 2017).

No caso apresentado, radiograficamente podemos ver regiões com múltiplas massas radiopacas, acometendo bilateralmente o osso mandibular, indo de acordo com as referências bibliográficas pesquisadas. As lesões coincidem com áreas de ausência dentária da mandíbula, tomando como hipótese que o desenvolvimento dessa lesão tenha sido alavancado pelas exodontias dos molares.

Segundo Cavalcante PHP, et al. (2018), à utilização de exames de imagens como a tomografia computadorizada de feixe cônico pode ser considerada um importante recurso para o diagnóstico diferencial, uma vez que apresentará áreas hiperdensas ou de densidade mista, além de expansão óssea e afinamento das placas corticais, características comuns a patologia em questão. Associando-se esses achados imaginológicos aos clínicos, o profissional chegará ao correto diagnóstico e posteriormente uma assertiva conduta terapêutica.

No que se refere a conduta terapêutica da DCOF, para casos assintomáticos não se faz necessária a realização de intervenções, apenas de avaliações periódicas clínicas e radiográficas. Já nos casos sintomáticos, o tratamento invasivo é necessário quando há infecção local associada (AMARISTA-ROJAS FJ, et al., 2016; COSTA FWG, et al., 2018; DAVIET-NOUAL V, et al., 2017; YILDIRIM E, et al., 2016).

Devido ao bom estado de saúde da paciente, seu desejo pela reabilitação protética e sua sintomatologia e protocolo da instituição, foi necessário o tratamento cirúrgico para remoção da lesão. A paciente encontrase em acompanhamento periódico através de exames clínicos e radiográficos à cada 06 (seis) meses, até dado momento não houve indício de recidiva da lesão. 
Enfatiza-se com este caso clínico apresentado a importância de saber as características que as lesões fibro-ósseas possuem, sendo de fundamental importância o conhecimento do Cirurgião Dentista tenha sobre as características clínicas e radiográficas da DCOF, pois baseado na correlação da anamnese, achados clínicos, características imaginológicas e histopatológicas, será estabelecido uma adequada conduta clínica. Dessa forma, apesar do estudo ser limitado apenas a um caso clínico, espera-se que esse estudo contribua como base para tomada de decisões terapêuticas pela comunidade odontológica.

\section{REFERÊNCIAS}

1. AMARISTA-ROJAS FJ, et al. Displasia ósea florida: reporte de um caso y revisión de la literatura. Rev. Odont. Mex. México, 2016; 20(2): 123-124.

2. BRODY A, et al. Difficulties in the diagnosis of periapical translucencies and in the classification of cemento-osseous dysplasia. BMC Oral Health. 2019; 19(1):139.

3. CAPELLA LRC, OLIVEIRA RJ. Atlas de Radiografia Panorâmica para o Cirurgião-Dentista. São Paulo: Editora Santos, 2014; $418 \mathrm{p}$.

4. CARVALHO CHP, et al. Florid cemento-osseous dysplasia and osteomyelitis: a case report of a simultaneous presentation. Rev. odonto ciênc. Porto Alegre, 2012; 27(2): 166-169.

5. CASTRO TF, et al. Manifestações imaginológicas distintas na displasia cemento-óssea florida, RFO. Passo Fundo, 2017; 22(2): 203-206

6. CAVALCANTE PHP, et al. Cemento-Osseous Dysplasias: Imaging Features Based on Cone Beam Computed Tomography Scans. Braz. Dent. J., Ribeirão Preto, 2018; v. 29(1), p: 99-104.

7. COSTA FWG, et al. Importância dos aspectos imaginológicos no plano de tratamento da displasia óssea florida: relato de caso. Rev. Cir. Traumatol. Buco-Maxilo-Fac. Camaragibe, 2018; 18(3): 26-30.

8. DAVIET-NOUAL V, et al. Differentiating early stage florid osseous dysplasia from periapical endodontic lesions: a radiological-based diagnostic algorithm. BMC oral health. Bethesda, 2017;17(1): 161.

9. EL-NAGGAR AK, et al. WHO classification of head and neck tumours. 4 ed. Lyon: IARC, 2017.

10. KATO CNAO, et al. Retrospective study of 383 cases of fibro-osseous lesions of the jaws. J. Oral Maxillo fac. Surg. 2018; 76(11): 2348-2359.

11. LIMA ALC, et al. Displasia cemento-óssea florida: relato de caso. REAS/EJCH. 2019; 20(20): 560-564

12. MACDONALD DS. Maxillofacial fibro-osseous lesions. Clin. Radiol. 2015; 70(1): 25-36.

13. NEVILLE BW, et al. Patologia óssea: dysplasia cemento-óssea florida. In: NEVILLE, B. W. et al. Patologia oral e Maxilofacial. 4 ed. Rio de Janeiro: Guanabara. Koogan, 2016. 871 p.

14. OLIVEIRA CNA. Epidemiologia das lesões fibro-ósseas benignas dos maxilares, MG. Dissertação (Mestrado em Estomatologia) - Universidade Federal de Minas Gerais, Faculdade de Odontologia. Minas Gerais, 2016 ; 102 p.

15. PAPAIZ EG, et al. Atlas de Tomografia Computadorizada por Feixe Cônico para o Cirurgião dentista. São Paulo: Editora Santos, 2011; 1152 p.

16. PEREIRA DL. Avaliação clínica e radiográfica de pacientes afetados por displasia óssea florida - estudo retrospectivo e multicêntrico colaborativo, SP. Dissetação (Mestrado em Estomatopatologia) - Universidade Estadual de Campinas, Faculdade de Odontologia de Piracicaba. São Paulo, 2016; 59 p.

17. SARMENTO DJS, et al. Severe florid cemento-osseous dysplasia: a case report treated conservative lyand literature review. Oral Maxillofac. Surg. 2013; 7(1): 43-46.

18. SHAH S, et al. Achados de TC de acompanhamento de cementoma gigante familiar recorrente de uma criança do sexo feminino. Skeletal Radiol 2012; 41: 341-6.

19. SOUZA JÚNIOR EJC, et al. Displasia cemento-óssea florida: relato de caso. Odontol Clín Científ. 2008 ; 7: 347 - 51.

20. YILDIRIM E, et al. Floridcemento-osseousdysplasia: a rare case report evaluated with cone-beam computed tomography. J. Oral Maxillofac. Pathol. 2016; 20(2); 329-332. 